\title{
Prevalence, risk-inducing lifestyle, and perceived susceptibility to kidney diseases by gender among Nigerians residents in South Western Nigeria
}

\author{
Monica Ewomazino Akokuwebe ${ }^{1}$, Clifford Odimegwu', Femi Omololu ${ }^{2}$
}

1. University of the Witwatersrand, Demography and Population Studies Programme.

2. University of Ibadan Faculty of Social Sciences, Sociology.

\begin{abstract}
Background: Kidney disease (KD) is increasingly recognized as a major public health problem worldwide with rising incidence and prevalence. Early identification of $\mathrm{KD}$ risk factors will slow down progression to kidney failure and death.

Objective: To determine the prevalence, risk-inducing lifestyle and perceived susceptibility among Nigerians in South-western Nigeria.

Methods: A pretested structured questionnaire was employed to draw information on socio-demographic, knowledge, risk-inducing lifestyle and perceived susceptibility to conventional risk factors of $\mathrm{KD}$ from 1757 residents aged $\geq 15$ years.

Results: The mean age of the respondents was $47.61 \pm 13.0$ years with a male-female ratio of 1.13:1. Knowledge of KD was low (mean score 2.29; 95\% CI: 2.18, 2.32). The prevalence of some established KD risk factors was regular use of herbal medications, $26.8 \%$ and physical inactivity, $70.0 \%$. Females with factors such as use of herbal drink [RRR: 1.56; CI=1.062.30; $\mathrm{p}=0.02$ ] and smoking [RRR: $2.72 ; \mathrm{CI}=1.37-5.37 ; \mathrm{p}=0.00$ ] predicted increased odds of perceived susceptibility to KD than their male counterparts.

Conclusion: The prevalence of KD risk-inducing lifestyles was high. More emphasis should be placed on effective public health programmes towards behavioural change in order to adopt lifestyle modification as well as to reduce the tendency to develop KD.

Keywords: Kidney disease; risk-inducing lifestyles; perceived susceptibility.

DOI: https://doi.org/10.4314/ahs.v20i2.40

Cite as: Akokunvebe ME, Odimegwn C, Omololu F. Prevalence, risk-inducing lifestyle, and perceived susceptibility to kidney diseases by gender among Nigerians residents in South Western Nigeria. Afri Health Sci. 2020; 20(2): 860-870. bttps:// doi.org/10.4314/abs.v20i2.40
\end{abstract}

\section{Introduction}

The rising incidence and prevalence of kidney disease $(\mathrm{KD})$ is worldwide and contributes significantly to morbidity, mortality and decreased life expectancy of affected individuals, particularly with the poorest populations at the highest risk $^{1,2,3}$, posing great challenges for the affected persons, their families and the nation as a whole. The burden appears to be more evident in sub-Saharan Africa4 as it tends to affect middle-aged, elderly populations as well as relatively younger individuals in their prime and economically productive age cohorts $^{4,5}$. Data from developed countries revealed that

\section{Corresponding author:}

Monica Ewomazino Akokuwebe,

University of the Witwatersrand,

Demography and Population Studies Programme

Tel: +27670020681

Email: monica.akokuwebe@gmail.com one out of eight adult Americans disclose evidence of $\mathrm{KD}^{6}$ and comparable estimates have been reported in Asia countries ${ }^{7,8}$. Similar figures are lacking in Africa, including in Nigeria, owing to dearth of national registries of $\mathrm{KD}$ and limited surveys. Although, epidemiological and community-based studies in Nigeria have reported chronic kidney disease (CKD) incidence between 1.6\% and $12.4 \% 16$ and prevalence of $11.4-18.8 \% 0^{17,18}$.

Studies ${ }^{9-11}$ have been conducted in different regions of Nigeria, with unpredictable outcomes to enumerate the magnitude of KD burden and its primary risk factors. Most of these studies were hospital-based. Their characterization of chronicity was based on duration of typical signs and symptoms inclining towards $\mathrm{KD}$ advanced stages ${ }^{12,9}$. These hospital-based studies fail to include individuals who lack access to healthcare ${ }^{13,14}$. Other factors contributing to KD burden in Nigeria include poor literacy level, inadequate knowledge of risk factors, dearth of KD prevention programmes, poor access to 
healthcare, late presentation, limited renal replacement therapy and its unaffordability, as well as harmful socio-cultural practices ${ }^{15,3}$. Previous studies ${ }^{15-18,3}$ carried out in different parts of Nigeria revealed lifestyle risk factors such as age, elevated blood pressure (BP), presence of diabetes mellitus, obesity, habitual intake of analgesics and herbs associated with KD development or progression. Other risk factors like sedentary living and physical inactivity were documented as becoming prevalent among certain populations, predisposing them to complications such as obesity, and unmanaged hypertension, by extension, KD progression ${ }^{19,11}$. This situation could have been caused by low awareness and inadequate knowledge as well as poor perception, as noted in many Nigerian studies ${ }^{16-17,3,11}$. Thus, knowledge affects informed decision towards $\mathrm{KD}$ ailments and medical knowledge of $\mathrm{KD}$ health condition will inform an individual's action towards preventive strategies as well as risk factors modifications.

And since lifestyle and environmental factors influences major risk factors and causes of $\mathrm{KD}$, population-based preventive strategies appear to be the cheapest and best solution to address KD burden. Emphasis on health risk awareness are likely to improve health behaviour of individuals and increase their risk perception as well as making themselves available for screening and early diagnosis ${ }^{3,11}$. With early identification of KD risk factors and diagnosis among individuals, its associated morbidity and mortality with its significant economic and public health burden will be reduced. No community-based study on prevalence, risk-inducing lifestyles and perceived susceptibility to KD by gender has been carried out in Lagos State to the best of the authors' knowledge. The aim of this study was to determine the prevalence and risk-inducing lifestyles as well as to evaluate predictors of perceived susceptibility to $\mathrm{KD}$ risk among Nigerians resident in Lagos State. This study adds to the body of knowledge in this area.

\section{Methods}

\section{Study design and population}

This was a cross-sectional study conducted in six urban and two rural local government areas (LGAs) of Lagos State, South-west Nigeria. The six urban LGAs were Lagos Island, Lagos Mainland, Ajeromi-Ifelodun, Somolu, Kosofe and Agege; while Epe and Ikorodu were the two rural LGAs. Lagos State was selected for this study owing to the fact that it featured prominently as one of the kidney-prone states in $2015^{20}$. Respondents who were 15 years or older constituted the study population.
A previous community-based study in Ekiti and Osun States of South-west Nigeria by Oluyombo et al. obtained a CKD prevalence of $14.2 \%{ }^{21}$. The sample size for this study was extrapolated from that value, with 95\% confidence and 5\% error margin. Based on the appropriate sample size formula for study population greater than $10,000^{22,21}$, this produced one thousand eight hundred $(1,800)$ participants. This number was increased to one thousand eight hundred and fifty $(1,850)$ to make allowance for possible non-responders and to increase the scope of the study. Informed and assent consent forms were taken from each of the respondents who agreed to participate in the study. The respondents who were $<15$ years, did not take part in the study; non-Nigerians as well as non-permanent residents in Lagos State were excluded from the study.

\section{Sampling method}

The multistage systematic sampling technique was used. According to the National Population Commission (NPC) of Nigeria Census of 2006, the estimated population of Lagos State, projected to 2016, was $12,479,240^{23}$, using the projection formula. There are 75 selected urban and 38 rural enumeration areas (EAs), totalling 113 EAs in the eight sampled urban and rural LGAs, which served as the sampling units for the purpose of this study. Twenty-four EAs (representing 39\% of the total EAs) were selected randomly by balloting. The population of the respondents from 15 years and above in the selected EAs was estimated using the NPC figures of 2006 projected to 2016 and estimated number of households at 6.5 persons. This gave 421,757. Dividing this estimated population $(421,757)$ by the sample size $(1,850)$ gave the sampling interval for systematic selection of participants, which was approximately 1:10. Hence one in every 10th respondents was given exclusive numbers for identification. The Kish conversion sheet was used to select the respondents for the study in each household ${ }^{24}$. In situations where the selected EAs were not adjacent, the first house in the next EA was regarded as an extension of the previous EA and the selection procedure continued.

\section{Study procedure}

The methodology used in this study was based on the KDIGO 2017 clinical practice guideline for the evaluation and management of CKD's recommended STEPS approach to KD surveillance with some modifications ${ }^{1}$, which focuses on topics related to the diagnosis, evaluation, prevention and treatment of CKD. This is a simple, standardized guideline for collecting, analyzing and disseminating data from numerous stakeholders, includ- 
ing patients, subject matter experts, and industry and national organizations. The guidelines approach focuses on obtaining core data on the established risk factors that determine the major disease burden and is designed to help countries build and strengthen their surveillance capacity. The STEPS instrument covers four different levels or 'steps' of risk factors assessment. These steps are questionnaire, physical measurements and biochemical measurements. Estimation of perceived susceptibility and psychological measurements was not part of the original STEPS instrument but was added in this study to correctly define the outcome variable of interest (perceived susceptibility to KD risk). This constituted a modification of to the original STEPS instrument.

The study was carried out in two phases. In the pilot phase, the study was pre-tested on ten percent of the sample size in a different enumeration area (EA). In the second phase, health talk was delivered at eight different community town halls in the EAs selected for the study. Attendees were informed of the study and those residing in the selected houses were expected to willingly participate in the study. A pre-tested semi-structured questionnaire was administered to each participant by a trained research assistant. Information about age, occupation, education, sex, marital status, income, religion, and risk-inducing lifestyles (alcohol, smoking, use of medicines without prescription, use of analgesics, sedentary living, and consumption pattern of poor diets) was obtained. The study commenced on 14 June 2016 and ended on 30 September 2016.

\section{Definitions}

Older age group was regarded as over 35 years in both sexes according to the definition given by Amarya et al. in developed countries ${ }^{24}$. For the purpose of this study, individuals with significant intake of herbal supplements or traditional drink were regarded as having any history of weekly intake of herbal supplement or traditional herb preparation for one month or more either currently or in the past. Analgesic abuse was regarded as cumulative lifetime use of more than 5000 pills of analgesics ${ }^{25}$. This was calculated from multiplying the average number of pills consumed in a week by the duration of use in years. Heavy smokers was regarded as those who smoke greater than or equal to 25 or more cigarettes a day ${ }^{26}$. The knowledge of $\mathrm{KD}$ was by a positive response to a question "Have you heard of KD before?" Knowledge and beliefs about KD were assessed by asking about the location, number and functions of the kidney, signs and symptoms of $\mathrm{KD}$ and knowledge of the risk factors. Using a calculated composite score for knowledge ranging from 0-28 of the knowledge questions, each item was first scored as true (1) or false (0) with the responses 'I do not know' and 'I am not very sure' as false. Then, the sum of the 28 scored items were obtained and composite scores were reported as mean ( $95 \%$ confidence interval [CI]) with overall weighted mean knowledge score of 3.33 (95\% CI 2.92, 3.73). Similarly, for binary logistic regression for demographic predictors of KD knowledge by gender distribution, participants' knowledge was scored arbitrarily as good or poor based on the proportion of total score (28) and $\geq 14 \%$ of 28 questions $(=\geq 50 \%$ ) was good while $<14 \%$ of 28 questions $(=<50 \%)$ was poor knowledge. Also, perceived susceptibility to $\mathrm{KD}$ risk was assessed with perception questions with total score of 60 . However, perceived susceptibility was by a positive response to the question "Given your present lifestyles, how susceptible do you think you are to KD?" Perceived susceptibility to KD risk was categorized into three levels, namely: low, intermediate and high. Score $<15 \%$ was low, $15 \%-29 \%$ was intermediate and $\geq 30 \%$ was high perceived susceptibility to KD risk. For the multinomial logistic regression, the respondents with no formal schooling were classified as non-educated, while those with primary, secondary and university schooling were classified as educated for the purpose of this study.

\section{Ethical consideration}

The study was approved by the Social Sciences and Humanities Research Ethics Committee (SSHEC) University of Ibadan (ref. no. UI/SSHEC/14/0003) and was conducted in compliance with the Helsinki Declaration and ethical consideration procedures. Informed and assent written consent forms were obtained from the participants where appropriate.

\section{Statistical methods}

Data were analyzed using SPSS version 22 (IBM SPSS Statistics for Windows, Version 22.0. Armonk, NY: IBM Corp.) Data were presented as mean \pm standard deviation and categorical variables were compared using Chi-square and contingency tables. Multivariate binary logistics regression using variables whose univariate analysis was significant was used to estimate the independent association between $\mathrm{KD}$ risk-inducing lifestyles and gender distribution. The multinomial logistics regression was used to estimate and predict the relationship between perceived susceptibility and risk-inducing lifestyles in addition to demographic factors. The level of significance was set at $\mathrm{p} \leq 0.05$. 


\section{Results}

One thousand, eight hundred and fifty respondents were seen during the study period of whom one thousand seven hundred and fifty-seven had complete data. Table 1 shows the respondents' socio-demographic characteristics; which include 932 males and 825 females with a male-female ratio of 1.9:2. The peak age was $35+$ years, with male mean age of $47.61 \pm 13.0$ years and female mean age of $33.45 \pm 11.5$ years respectively, with an overall mean age of $34.15 \pm 10.6$ years. The median age for male respondents was 50 years and 34 years for female respondents with an overall median age of 42.5 years (range $15-85+$ ). The overall weighted mean knowledge score was 3.33 (95\% CI 2.92, 3.73) out of twenty-eight possible points and males had a higher mean knowledge score compared to females (Table 1).

Table 1: Characteristics of study participants (Data are expressed as mean \pm standard deviation, ratio, number, percentage and Chi-square)

\begin{tabular}{|c|c|c|c|}
\hline Variables & Male $n=932 ; n(\%)$ & Female $n=825 ; n(\%)$ & Total= 1757; N (\%) \\
\hline Mean age \pm s.d. (years) & $47.61 \pm 13.0$ & $33.45 \pm 11.5$ & $34.15 \pm 10.6$ \\
\hline Median age & 50 years & 34 years & 42.5 years \\
\hline Male to female ratio & & & $1.9: 2$ \\
\hline $\begin{array}{l}\text { Mean Knowledge score }(95 \% \\
\text { CI) }\end{array}$ & $2.45(2.14,3.23)$ & $2.12(2.08,2.28)$ & $2.29(2.18,2.32)$ \\
\hline $\begin{array}{l}\text { Age } \\
15-24 \text { years } \\
25-34 \text { years } \\
35^{+} \text {years } \\
\end{array}$ & $\begin{array}{l}253(27.1) \\
243(26.1) \\
436(46.8)\end{array}$ & $\begin{array}{l}239(29.0) \\
230(27.9) \\
356(43.2)\end{array}$ & $\begin{array}{l}492(28.0) \\
473(25,9) \\
792(45.1) \\
\end{array}$ \\
\hline $\begin{array}{l}\text { Educational Level } \\
\text { No schooling } \\
\text { Primary } \\
\text { Secondary and Above }\end{array}$ & $\begin{array}{c}26(2.8) \\
81(8.7) \\
825(88.5)\end{array}$ & $\begin{array}{c}20(2.4) \\
53(6.4) \\
752(91.2)\end{array}$ & $\begin{array}{c}46(2.6) \\
134(7.6) \\
1577(89.8)\end{array}$ \\
\hline $\begin{array}{l}\text { Ethnic group } \\
\text { Yoruba } \\
\text { Igbo } \\
\text { Hausa } \\
\text { Others } \\
\end{array}$ & $\begin{array}{c}527(56.5) \\
246(26.4) \\
64(6.9) \\
95(10.2) \\
\end{array}$ & $\begin{array}{c}432(52.4) \\
257(31.2) \\
55(6.7) \\
81(9.8) \\
\end{array}$ & $\begin{array}{c}959(54.6) \\
503(28.6) \\
119(6.8) \\
176(10.0) \\
\end{array}$ \\
\hline $\begin{array}{l}\text { Marital Status } \\
\text { Single } \\
\text { Married } \\
\text { Previously married }\end{array}$ & $\begin{array}{c}546(58.6) \\
352(37.8) \\
34(3.6) \\
\end{array}$ & $\begin{array}{c}441(53.5) \\
326(39.5) \\
58(7.0) \\
\end{array}$ & $\begin{array}{c}987(56.2) \\
678(38.6) \\
92(5.2) \\
\end{array}$ \\
\hline $\begin{array}{l}\text { Religion } \\
\text { Christianity } \\
\text { Islam } \\
\text { Traditionalist }\end{array}$ & $\begin{array}{c}673(72.2) \\
237(25.4) \\
22(2.4) \\
\end{array}$ & $\begin{array}{c}603(73.1) \\
193(23.4) \\
29(3.5) \\
\end{array}$ & $\begin{array}{c}1276(72.6) \\
430(24.5) \\
51(2.9) \\
\end{array}$ \\
\hline $\begin{array}{l}\text { Employment status } \\
\text { Not working } \\
\text { Working }\end{array}$ & $\begin{array}{l}201(21.6) \\
731(78.4)\end{array}$ & $\begin{array}{l}240(29.1) \\
585(70.9)\end{array}$ & $\begin{array}{c}441(25.1) \\
1316(74.9)\end{array}$ \\
\hline $\begin{array}{l}\text { Monthly Income } \\
\text { Low } \\
\text { Middle } \\
\text { High } \\
\end{array}$ & $\begin{array}{l}267(28.6) \\
225(24.1) \\
440(47.2)\end{array}$ & $\begin{array}{l}315(38.2) \\
207(25.1) \\
303(36.7)\end{array}$ & $\begin{array}{l}582(33.1) \\
432(24.6) \\
743(42.3)\end{array}$ \\
\hline $\begin{array}{l}\text { Setting } \\
\text { Urban } \\
\text { Rural }\end{array}$ & $\begin{array}{l}696(74.7) \\
236(25.3)\end{array}$ & $\begin{array}{l}624(75.6) \\
201(24.4)\end{array}$ & $\begin{array}{l}1320(75.1) \\
437(24.9)\end{array}$ \\
\hline
\end{tabular}

p-value is significant at 0.05

Table 2 shows the profile of self-reported risk-inducing lifestyles by gender distribution. The significant and commonest risk-inducing lifestyles reported were lack of daily activities $(74.9 \%)$, use of herbal medications $(26.8 \%)$, use of traditional herbal drink (46.4\%), chronic ingestion of alcohol (33.2\%), smoking (11.5\%), physical inactivity $(70.0 \%)$ and sedentary living $(29.0 \%)$ as well as consumption pattern of poor diets (low vegetables $(75.8 \%)$, low fruits $(64.9 \%)$ and high processed dairy foods $(48.4 \%)$. 
Table 2: Profile of self-reported risk-inducing lifestyles by gender distribution (univariate analysis $) ;(\mathrm{P}<0.005)$

\begin{tabular}{|c|c|c|c|c|c|c|}
\hline Variables & $\begin{array}{c}\text { Male, } \mathbf{n}=932 \\
\mathbf{n}(\%)\end{array}$ & Female, $n=825, n(\%)$ & Total $=1757, \mathrm{~N}(\%)$ & $* * \mathbf{O R}$ & $95 \% \mathrm{CI}$ & p-value \\
\hline $\begin{array}{l}\text { Age (years) } \\
<30 \\
\geq 30\end{array}$ & $\begin{array}{l}339(36.4) \\
593(63.6)\end{array}$ & $\begin{array}{l}331(40.1) \\
494(59.9)\end{array}$ & $\begin{array}{c}670(38.1) \\
1087(61.9)\end{array}$ & 1.17 & $0.97-1.42$ & 0.11 \\
\hline $\begin{array}{l}\text { Daily Activities } \\
\text { Yes } \\
\text { No } \\
\end{array}$ & $\begin{array}{l}731(78.4) \\
201(21.6) \\
\end{array}$ & $\begin{array}{l}585(70.9) \\
240(29.1) \\
\end{array}$ & $\begin{array}{c}1316(74.9) \\
441(25.1) \\
\end{array}$ & 0.67 & $0.54-0.83$ & $0.00^{*}$ \\
\hline $\begin{array}{l}\text { Misuse of } \\
\text { medicines } \\
\text { Yes } \\
\text { No }\end{array}$ & $\begin{array}{c}89(9.5) \\
843(90.5)\end{array}$ & $\begin{array}{c}65(7.9) \\
760(92.1)\end{array}$ & $\begin{array}{c}154(8.8) \\
1603(91.2)\end{array}$ & 0.810 & $0.58-1.13$ & 0.24 \\
\hline $\begin{array}{l}\text { Medicines without } \\
\text { prescription } \\
\text { Yes } \\
\text { No } \\
\end{array}$ & $\begin{array}{l}297(31.9) \\
635(68.1)\end{array}$ & $\begin{array}{l}241(29.2) \\
584(70.8)\end{array}$ & $\begin{array}{c}538(30.6) \\
1219(69.4)\end{array}$ & 0.88 & $0.72-1.08$ & 0.23 \\
\hline 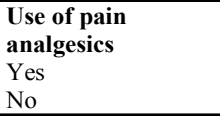 & $\begin{array}{l}284(30.5) \\
648(69.5)\end{array}$ & $\begin{array}{l}219(26.5) \\
606(73.5)\end{array}$ & $\begin{array}{c}503(28.6) \\
1254(71.4)\end{array}$ & 0.83 & $0.67-1.02$ & 0.07 \\
\hline $\begin{array}{l}\text { Use of herbal } \\
\text { medications } \\
\text { Yes } \\
\text { No } \\
\end{array}$ & $\begin{array}{l}271(29.1) \\
661(70.9)\end{array}$ & $\begin{array}{l}200(24.2) \\
625(75.8)\end{array}$ & $\begin{array}{c}471(26.8) \\
1286(73.2)\end{array}$ & 0.78 & $0.63-0.97$ & $0.02 *$ \\
\hline $\begin{array}{l}\text { Use of traditional } \\
\text { herb drink } \\
\text { Yes } \\
\text { No } \\
\end{array}$ & $\begin{array}{l}481(51.6) \\
461(48.4)\end{array}$ & $\begin{array}{l}335(40.6) \\
490(59.4)\end{array}$ & $\begin{array}{l}816(46.4) \\
941(53.6)\end{array}$ & 0.64 & $0.53-0.78$ & $0.00^{*}$ \\
\hline $\begin{array}{l}\text { Chronic ingestion } \\
\text { of alcohol } \\
\text { Yes } \\
\text { No } \\
\end{array}$ & $\begin{array}{l}408(43.8) \\
524(56.2)\end{array}$ & $\begin{array}{l}176(21.3) \\
649(78.7)\end{array}$ & $\begin{array}{c}584(33.2) \\
1173(66.8)\end{array}$ & 0.35 & $0.28-0.43$ & $0.00 *$ \\
\hline $\begin{array}{l}\text { Heavy smokers } \\
\text { Yes } \\
\text { No } \\
\end{array}$ & $\begin{array}{l}157(16.8) \\
775(83.2) \\
\end{array}$ & $\begin{array}{c}45(5.5) \\
780(94.5) \\
\end{array}$ & $\begin{array}{c}202(11.5) \\
1555(88.5) \\
\end{array}$ & 0.26 & $0.20-0.40$ & $0.00 *$ \\
\hline $\begin{array}{l}\text { Physical inactivity } \\
\text { Yes } \\
\text { No }\end{array}$ & $\begin{array}{l}687(73.7) \\
245(26.3) \\
\end{array}$ & $\begin{array}{l}543(65.8) \\
282(34.2) \\
\end{array}$ & $\begin{array}{c}1230(70.0) \\
527(30.0) \\
\end{array}$ & 0.69 & $0.56-0.84$ & $0.00^{*}$ \\
\hline $\begin{array}{l}\text { Sedentary living } \\
\text { Yes } \\
\text { No } \\
\end{array}$ & $\begin{array}{l}250(26.8) \\
682(73.2) \\
\end{array}$ & $\begin{array}{l}276(33.5) \\
549(66.5) \\
\end{array}$ & $\begin{array}{c}526(29.9) \\
1231(70.1) \\
\end{array}$ & 1.37 & $1.12-1.68$ & $0.00^{*}$ \\
\hline $\begin{array}{l}\text { Poor diet } \\
\text { Lack of vegetables } \\
\text { \& legumes } \\
\text { Yes } \\
\text { No } \\
\end{array}$ & $\begin{array}{l}680(73.0) \\
252(27.0)\end{array}$ & $\begin{array}{l}651(78.9) \\
174(21.1)\end{array}$ & $\begin{array}{c}1331(75.8) \\
426(24.2)\end{array}$ & 1.39 & $1.11-1.73$ & $0.00 *$ \\
\hline $\begin{array}{l}\text { Lack of fruits } \\
\text { Yes } \\
\text { No } \\
\end{array}$ & $\begin{array}{l}581(62.3) \\
351(37.7)\end{array}$ & $\begin{array}{l}559(67.8) \\
266(32.2)\end{array}$ & $\begin{array}{c}1140(64.9) \\
617(35.1)\end{array}$ & 1.27 & $1.04-1.55$ & $0.02 *$ \\
\hline $\begin{array}{l}\text { High in grain foods } \\
\text { Yes } \\
\text { No } \\
\end{array}$ & $\begin{array}{l}778(83.5) \\
154(16.5) \\
\end{array}$ & $\begin{array}{l}659(79.9) \\
166(20.1) \\
\end{array}$ & $\begin{array}{c}1437(81.8) \\
320(18.2) \\
\end{array}$ & 0.79 & $0.62-1.00$ & 0.05 \\
\hline $\begin{array}{l}\text { High in processed } \\
\text { meats } \\
\text { Yes } \\
\text { No } \\
\end{array}$ & $\begin{array}{l}729(78.2) \\
203(21.8)\end{array}$ & $\begin{array}{l}640(77.6) \\
185(22.4)\end{array}$ & $\begin{array}{c}1369(77.9) \\
388(22.1)\end{array}$ & 0.96 & $0.77-1.21$ & 0.75 \\
\hline $\begin{array}{l}\text { High in processed } \\
\text { dairy foods } \\
\text { Yes } \\
\text { No } \\
\end{array}$ & $\begin{array}{l}426(45.7) \\
506(54.3)\end{array}$ & $\begin{array}{l}424(51.4) \\
401(48.6)\end{array}$ & $\begin{array}{l}850(48.4) \\
907(51.6)\end{array}$ & 1.26 & $1.04-1.51$ & $0.02 *$ \\
\hline 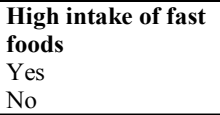 & $\begin{array}{l}245(26.3) \\
687(73.7\end{array}$ & $\begin{array}{l}237(28.7) \\
588(71.3)\end{array}$ & $\begin{array}{c}482(27.4) \\
1275(72.8)\end{array}$ & 1.13 & $0.92-1.39$ & 0.25 \\
\hline
\end{tabular}

As regards demographic predictors of $\mathrm{KD}$ knowledge by gender distribution, primary education $(\mathrm{OR}=13.33$, $\mathrm{CI}=4.55-39.08, \quad \mathrm{p}<0.05), \quad$ secondary+ education $(\mathrm{OR}=2.61, \mathrm{CI}=1.67-4.14, \mathrm{p}<0.05)$ and rural setting $(\mathrm{OR}=1.42, \mathrm{CI}=1.03-1.97, \mathrm{p}<0.05)$ significantly predicted increased odds of $\mathrm{KD}$ knowledge among male respondents more than their female counterparts (Table 3). Primary (OR=4.09, $\mathrm{CI}=1.61-10.37, \mathrm{p}<0.05)$ and secondary/university education $(\mathrm{OR}=3.63, \mathrm{CI}=2.04$ -
6.46, $\mathrm{p}<0.05)$ were found to have higher odds of $\mathrm{KD}$ knowledge among the female respondents than their male counterparts (Table 3). Moreover, Fig 1 captures the distribution of respondents' perceived susceptibility to KD risks by gender. Both male and female respondents were found to have low perceived susceptibility to $\mathrm{KD}$ risk, with slight increase among the female respondents. However, 586 did not report their level of perceived susceptibility to $\mathrm{KD}$ risk. 
Table 3: Binary logistic regression of demographic predictors of KD knowledge by gender distribution

\begin{tabular}{|c|c|c|c|c|c|c|c|c|c|c|}
\hline \multicolumn{6}{|c|}{ Males } & \multicolumn{5}{|c|}{ Females } \\
\hline Variables & $\begin{array}{c}\text { Coefficient } \\
(\beta)\end{array}$ & $\begin{array}{c}\begin{array}{c}\text { Standard } \\
\text { error }\end{array} \\
\end{array}$ & $\overline{\mathrm{OR}}$ & P-value & CI 95\% & $\begin{array}{c}\text { Coefficient } \\
(\beta)\end{array}$ & $\begin{array}{c}\text { Standard } \\
\text { error }\end{array}$ & $* * \mathbf{O R}$ & P-value & CI 95\% \\
\hline $\begin{array}{l}\text { Age (years) } \\
<30 \\
\geq 30\end{array}$ & -1.13 & 0.16 & 0.32 & $0.00 *$ & $0.23-0.44$ & -1.02 & 0.16 & 0.36 & $0.00 *$ & $0.26-0.50$ \\
\hline $\begin{array}{l}\text { Education } \\
\text { No schooling }\end{array}$ & & & & & & & & & & \\
\hline Primary & 2.59 & 0.55 & 13.33 & $0.00 *$ & $4.55-39.08$ & 1.41 & 0.48 & 4.09 & $0.00 *$ & $1.61-10.37$ \\
\hline $\begin{array}{l}\text { Secondary \& } \\
\text { Above }\end{array}$ & 0.96 & 0.24 & 2.61 & $0.00^{*}$ & $1.67-4.14$ & 1.29 & 0.29 & 3.63 & $0.00^{*}$ & $2.04-6.46$ \\
\hline $\begin{array}{l}\text { Employment } \\
\text { Not working } \\
\text { Working }\end{array}$ & -0.12 & 0.17 & 0.89 & 0.47 & $0.64-1.23$ & 0.18 & 0.16 & 1.20 & 0.28 & $0.87-1.65$ \\
\hline $\begin{array}{l}\text { Monthly } \\
\text { Income } \\
\text { Low }\end{array}$ & -1.19 & 0.17 & 0.31 & $0.00 *$ & $0.22-0.43$ & -1.10 & 0.18 & 0.33 & $0.00 *$ & $0.23-0.47$ \\
\hline $\begin{array}{l}\text { Middle } \\
\text { High }\end{array}$ & -0.28 & 0.18 & 0.76 & 0.13 & $0.53-1.08$ & -0.39 & 0.18 & 0.68 & $0.04 *$ & $0.47-0.97$ \\
\hline $\begin{array}{l}\text { Religion } \\
\text { Christianity } \\
\text { Islam }\end{array}$ & -0.12 & 0.47 & 0.89 & 0.80 & $0.36-2.21$ & -0.87 & 0.38 & 0.42 & $0.02 *$ & $0.20-0.89$ \\
\hline Traditionalist & 0.47 & 0.48 & 1.59 & 0.33 & $0.63-4.05$ & -0.44 & 0.40 & 0.65 & 0.28 & $0.30-1.42$ \\
\hline $\begin{array}{l}\text { Setting } \\
\text { Urban }\end{array}$ & & & & & & & & & & \\
\hline Rural & 0.35 & 0.17 & 1.42 & $0.03 *$ & $1.03-1.97$ & 0.07 & 0.17 & 1.08 & 0.67 & $0.77-1.51$ \\
\hline
\end{tabular}

*p-value is significant at $0.05 ; * * \mathrm{OR}=\mathrm{Odds}$ ratio; *** $\mathrm{OR}=\geq 1$ denotes good knowledge and $\mathrm{OR}=<1$ indicates poor knowledge

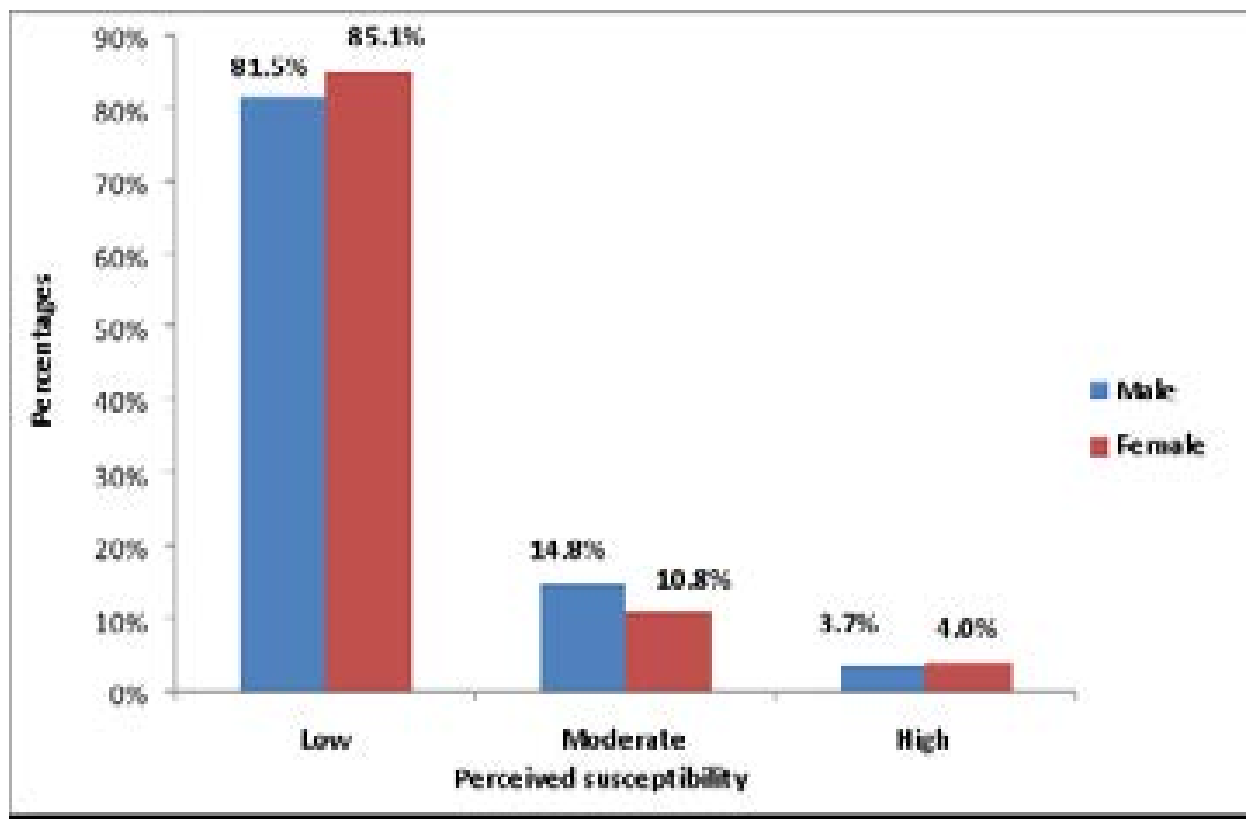

Figure 1: Respondents' perceived susceptibility to KD risk by gender

Table 4 shows the multinomial logistic regression analysis of adjusted independent predictors of perceived susceptibility to KD risk by gender distribution. The male respondents with either perceived intermediate or high susceptibility to $K D$ risk with age $\geq 30$ years [RRR:
1.12, $\mathrm{CI}=1.91-5.08, \mathrm{p}<0.05]$, use of traditional herbal drink [RRR: 1.87, $\mathrm{CI}=0.89-1.92, \mathrm{p}<0.05]$, chronic ingestion of alcohol [RRR: 1.84, CI=1.26-2.72, $\mathrm{p}<0.05$ ] and smoking [RRR: 2.41, CI=1.53-3.80, $\mathrm{p}<0.05]$ were found to be respectively $11 \%, 19 \%, 18 \%$, and $24 \%$ 
higher, predicting high $\mathrm{KD}$ risk. As regards the female 1.27, $\mathrm{p}<0.05]$ and use of traditional herbal drink [RRR: respondents, age $\geq 30$ years [RRR: $2.27, \mathrm{CI}=1.49-3.46,1.19, \mathrm{CI}=0.84-1.67, \mathrm{p}<0.05$ ] were respectively found to $\mathrm{p}<0.05$ ], use of pain analgesics [RRR: $1.18, \mathrm{CI}=0.55-$ be $23 \%, 12 \%$ and $12 \%$ higher predictors of $\mathrm{KD}$ risk (Table 4).

Table 4: Multinomial logistic regression analysis of risk-inducing lifestyles as predictors of perceived susceptibility to KD risk

\begin{tabular}{|c|c|c|c|c|c|c|c|c|c|c|}
\hline \multirow{4}{*}{$\begin{array}{l}\text { Perceived } \\
\text { Susceptibility } \\
\text { (base outcome) } \\
\text { Social } \\
\text { Characteristics }\end{array}$} & \multicolumn{5}{|c|}{ Male } & \multicolumn{5}{|c|}{ Female } \\
\hline & \multicolumn{2}{|c|}{ Intermediate } & \multicolumn{2}{|c|}{ High } & \multirow{2}{*}{ p-value } & \multicolumn{2}{|c|}{ Intermediate } & \multicolumn{2}{|c|}{ High } & \multirow{2}{*}{ p-value } \\
\hline & RRR & CI & RRR & CI & & RRR & CI & RRR & CI & \\
\hline & & & & & & & & & & \\
\hline $\begin{array}{l}\text { Educational } \\
\text { Level } \\
\text { Non-educated } \\
\text { Educated }\end{array}$ & 0.37 & $0.26-0.53$ & 0.52 & $0.33-0.82$ & $0.01^{*}$ & 0.53 & $0.35-0.82$ & 0.44 & $0.28-0.69$ & $0.00 *$ \\
\hline $\begin{array}{l}\text { Age (years) } \\
<30 \\
\geq 30 \\
\end{array}$ & 1.02 & $0.75-1.39$ & 1.12 & $1.91-5.08$ & $0.00^{*}$ & 1.79 & $1.26-2.55$ & 2.27 & $1.49-3.46$ & $0.00 *$ \\
\hline $\begin{array}{l}\text { Employment } \\
\text { status } \\
\text { Not working } \\
\text { Working }\end{array}$ & 0.93 & $0.64-1.35$ & 1.01 & $0.64-1.61$ & 0.70 & 1.11 & $0.78-1.59$ & 0.48 & $0.30-0.79$ & $0.00 *$ \\
\hline $\begin{array}{l}\text { Setting } \\
\text { Rural } \\
\text { Urban }\end{array}$ & 1.20 & $0.84-1.70$ & 1.57 & $1.03-2.39$ & $0.03 *$ & 2.22 & $1.53-3.22$ & 1.56 & $1.00-2.42$ & $0.05^{*}$ \\
\hline $\begin{array}{l}\text { Medicines } \\
\text { without } \\
\text { prescription } \\
\text { No } \\
\text { Yes }\end{array}$ & 0.61 & $0.17-1.18$ & 0.37 & $0.05-2.87$ & 0.34 & 1.08 & $0.21-5.60$ & 1.55 & $0.30-8.10$ & 0.60 \\
\hline $\begin{array}{l}\text { Use of pain } \\
\text { analgesics } \\
\text { No } \\
\text { Yes }\end{array}$ & 0.60 & $0.43-0.83$ & 0.61 & $0.40-0.91$ & $0.02 *$ & 1.81 & $0.55-1.17$ & 0.71 & $0.47-1.09$ & $0.04 *$ \\
\hline $\begin{array}{l}\text { Use of herbal } \\
\text { medications } \\
\text { No } \\
\text { Yes } \\
\end{array}$ & 1.07 & $0.77-1.49$ & 0.63 & $0.40-1.00$ & 0.05 & 0.80 & $0.54-1.19$ & 0.78 & $0.49-1.24$ & 0.29 \\
\hline $\begin{array}{l}\text { Use of } \\
\text { traditional } \\
\text { herbal drink } \\
\text { No } \\
\text { Yes }\end{array}$ & 1.87 & $1.37-2.55$ & 1.31 & $0.89-1.92$ & $0.00 *$ & 1.19 & $0.84-1.67$ & 1.56 & $1.06-2.30$ & $0.02 *$ \\
\hline $\begin{array}{l}\text { Chronic } \\
\text { ingestion of } \\
\text { alcohol } \\
\text { No } \\
\text { Yes }\end{array}$ & 1.51 & $1.11-2.06$ & 1.85 & $1.26-2.72$ & $0.00 *$ & 1.20 & $0.80-1.80$ & 1.37 & $0.87-2.15$ & 0.17 \\
\hline $\begin{array}{l}\text { Heavy smokers } \\
\text { No } \\
\text { Yes }\end{array}$ & 1.47 & $0.98-2.21$ & 2.41 & $1.53-3.80$ & $0.00 *$ & 0.81 & $0.34-1.93$ & 2.72 & $1.37-5.37$ & $0.00 *$ \\
\hline $\begin{array}{l}\text { Physical } \\
\text { inactivity } \\
\text { No } \\
\text { Yes }\end{array}$ & 0.84 & $0.58-1.21$ & 0.22 & $0.15-0.33$ & 0.34 & 0.60 & $0.422-0.86$ & 0.21 & $0.14-0.31$ & $0.00^{*}$ \\
\hline $\begin{array}{l}\text { Sedentary living } \\
\text { No } \\
\text { Yes }\end{array}$ & 0.70 & $0.49-1.00$ & 0.67 & $0.43-1.06$ & 0.09 & 1.63 & $1.15-2.30$ & 1.02 & $0.68-1.55$ & 0.92 \\
\hline $\begin{array}{l}\text { High intake of } \\
\text { fast foods } \\
\text { No } \\
\text { Yes }\end{array}$ & 0.82 & $0.58-1.16$ & 0.48 & $0.32-0.72$ & 0.26 & 0.74 & $0.51-1.076$ & 0.37 & $0.25-0.56$ & 0.12 \\
\hline
\end{tabular}

*p-value is significant at $0.05 ; * *$ Low perceived susceptibility was used as the reference category; ${ }^{* * *}$ RRR-relative risk ratio 


\section{Discussion}

This study assessed the prevalence and risk-inducing lifestyles as well as perceived susceptibility of Nigerians resident in South-western Nigeria regarding KD risk. Based on the findings, the prevalent $\mathrm{KD}$ risk-inducing lifestyles were use of herbal medications, use of traditional herbal drink, chronic ingestion of alcohol, smoking, physical inactivity and sedentary living among the respondents by gender distribution. This was lower among the female respondents than the male counterparts and the prevalence observed in some earlier studies in Nigeria did not tally with this study findings ${ }^{14,9,5}$. The disparity may be attributed to sex differences in dietary habits, age and lifestyles. Socio-cultural and economic factors which preferentially favour males in Nigerian communities may also contribute to their increased risk-inducing lifestyles.

Efforts should be made to screen individuals with risk factors for $\mathrm{KD}$ signs and the results should be comprehensively interpreted to allow an informed decision. The effect of avoiding risk factors on $\mathrm{KD}$ progression to chronic kidney disease as well as end-stage renal disease should be stressed, as the majority of affected victims could not afford the cost of dialysis or renal replacement therapy (RRT)27. High prevalence of KD among patients is as a result of the problem of lack of screening and early detection and treatment which contributed to KD burden. Therefore, KD development or progression can be delayed if detected early as sequelae of other preceding chronic ailments progression (for example hypertension, diabetes and cardiovascular disease).

In agreement with previous studies ${ }^{28,29}$, the respondents with adequate $\mathrm{KD}$ knowledge were found to indulge in risk-inducing lifestyles. This may be a reflection of $\mathrm{KD}$ denial or its associated risk factors and non-adherence to lifestyle modifications among the respondents, which are probable psychological and social aspects that could lead to poor health outcome and eventually natural progression of KD. Educational information should be tailored towards reduction of risk factors by adjusting lifestyle behaviour. For example, lifestyle behaviour such as use of medicines without medical doctors' prescription, herbal medications and traditional herbal drink should be discouraged. Nigerian studies have shown that herbal concoctions and medications are now a KD risk factor, which is one of the common causes of acute kidney injury $y^{30,12}$ with increasing morbidity and mortality of affected victims.
This study showed a significant relationship between education and KD knowledge. There are some studies which revealed that educational status is likely to influence individuals to seek for knowledge on health matters $^{3,10}$. The male respondents with education were found to have increased knowledge of KD risk-inducing lifestyles than their counterparts with no education. Knowledge of health matters influences an individual to avoid the risk factors and causes of KD development and progression. The reason for these apparent observations may be because knowledge affects informed decision towards KD ailments and such knowledge will guide the affected persons towards cues to actions or adopting preventive measures. Also, some studies directly linked poor awareness and knowledge of $\mathrm{KD}$ to $\mathrm{KD}$ development or progression ${ }^{16,3,10}$. Awareness facilitates easy communication that would lead to taking an informed decision and understanding of lifestyles modifications, early detection of $\mathrm{KD}$ and management, if one exists.

In addition, the demographic predictors of KD knowledge identified as an independent risk factors of $\mathrm{KD}$ among the respondents in this study were higher education, income, religion and urban setting. This is in line with the findings from previous studies that reported a positive association of demographic factors with $\mathrm{KD}$ risk $^{12,18}$. The above-mentioned demographic factors were positively associated with $\mathrm{KD}$ knowledge and its risk, but some other studies have contrary findings $\mathrm{s}^{31,32}$. The reason for these contradictory findings is not clear and so this contrary aspect demands further investigations.

Perceived susceptibility was low among the respondents by gender distribution, with slight increase among the female respondents. Factors such as age $\geq 30$ years, male gender, sedentary living and poor diet were independently associated with greater odds of low perceived susceptibility, and this is in agreement with earlier studies ${ }^{29,17,5}$. The implication of this is that as the population ages, there is need to intensify health education interventions and screening strategies to detect high-risk persons with $\mathrm{KD}$. The respondents with multiple risk factors, such as risk-inducing and bio-medical factors, had greater chances of KD occurrence or its progression. This corroborates previous hospital-based studies $^{14,17}$ and a similar study on misuse of medicines without medical doctors' prescription ${ }^{25}$. 
The respondents with lower perceived susceptibility were found to have increased risk-inducing lifestyles for $\mathrm{KD}$ risk. In reality, persons with adequate knowledge of $\mathrm{KD}$ and its risk factors should take precautionary measures towards KD risk. Low perceived susceptibility could be as a result of respondents not making themselves available for $\mathrm{KD}$ screening or health personnel are not communicating well $\mathrm{KD}$ and its risk factors to the general populace ${ }^{33}$. This is actually worth exploring in other studies to adequately have a first-hand information on how to stem the tide of CKD prevalence associated with increased morbidity and mortality of individuals.

Gender differences had a significant association with perceived susceptibility to $\mathrm{KD}$ risk in this study. This does not agree with the findings of many other studies, in which the male gender was reported to be a unmodifiable risk factor for $\mathrm{KD}^{29}$; while few studies have documented female prevalence of KD across Asia and Indian countries ${ }^{34,35}$. In particular, male gender with higher risk of $\mathrm{KD}$ may be attributed to their involvement in risky behaviours as well as in predisposing biomedical factors. Studies ${ }^{10,29}$ have revealed that actuals risks is being facilitated in controlling $\mathrm{KD}$ encouraging preventive actions for individuals at high risk.

However, the assumed impact of $\mathrm{KD}$ perceived risk is to stimulate behavioral modifications to prevent, detect, and manage $\mathrm{KD}$ in cases other than being in a condition of complying with stringent health requirements ${ }^{36,37}$. Thus, perceived risks are interconnected with impetus to act and to action, which have higher equal odds between perceived risks (beliefs) and actual risk (reality). Hence, individuals will understand the health implications of $\mathrm{KD}$ risk factors and begin to evaluate their personal health risks towards $\mathrm{KD}$.

Several hospital-based studies have reported an association between sedentary living with poor diets and perceived susceptibility to $\mathrm{KD}$ among $\mathrm{CKD}$-diagnosed patients ${ }^{29,36-38}$. On the contrary, a Nigerian study found an association in multivariate analysis between the presence of use of pain analgesics, use of herbal medications, chronic ingestion of alcohol and high intake of fast foods risk factors and gender distribution with respect to perceived susceptibility to KD risk ${ }^{3,39}$.

\section{Limitations}

The cross-sectional nature where respondents were visited only once during data collection and knowledge of $\mathrm{KD}$, was also self-reported. Also, in view of the cross-sectional nature of this study, further prospective studies about the association between perceived susceptibiity to $\mathrm{KD}$ risk and progression of kidney function could be done. The strengths of this study are the multi-stage random sampling method, and, to a large extent, a good coverage of the community where the study was carried out. Also, content and concurrent validity was employed in designing the knowledge and perception questions in order to validate this study outcomes ${ }^{3,14,16}$.

\section{Conclusion}

Knowledge of kidney diseases (KD) and prevalence of risk-inducing lifestyles was high among the male folks in the community, while perceived susceptibility by gender distribution was low. The independent risk factors for KD reported included old age, use of herbal medications, traditional herbal drink, chronic ingestion of alcohol and smoking. Based on the outcome of this study, we recommend that concerted effort should be made to improve the development of well-structured health education programmes in the community to produce a better understanding of $\mathrm{KD}$ burden and its related risk factors. Urgent attention needs to be paid to the risk factors for $\mathrm{KD}$ and to introduce preventive interventions to slow KD progression in Nigerian communities. Kidney disease should be discussed in the clinic with persons with multiple risk factors (lifestyles and biomedical factors). Screening programmes should incorporate more awareness talks on the disease, especially communities with limited knowledge of $\mathrm{KD}$ risk factors. For those diagnosed with $\mathrm{KD}$, dialysis and renal transplantation therapy should be encouraged in those that can support the treatment. Above all, preventive health programmes should be targeted in all grassroots' communities.

\section{Acknowledgements}

The supports of the DST-NRF Centre of Excellence (CoE) in Human Development, National Research Foundation and The William and Flora Hewlett Foundation for this research are acknowledged. Opinions expressed and conclusions arrived at are those of the authors, and are not necessarily to be attributed to the $\mathrm{CoE}$ in Human Development. The authors gratefully acknowledge the support of the Wits Demography and Population Studies Programme, School of Public Health and Social Sciences, Faculties of Health Sciences and Humanities, National Institute for the Humanities and Social Sciences and University of the Witwatersrand, Johannesburg, South Africa. 


\section{References}

1. National Kidney Foundation. KDIGO 2017 Clinical Practice Guideline for the Evaluation and Management of Chronic Kidney Disease. Kidney Int Suppl. 2017; 7 (1): 1-59.

2. Alebiosu CO, Ayodele OE. The global burden of chronic kidney disease and the way forward. Ethn Dis. 2005; 15 (3): 418-423.

3. Oluyombo R, Ayodele OE, Akinwusi PO, Okunola OO, Gbadegesin BA, Soje MO, Akinsola A. Awareness, knowledge and perception of chronic kidney disease in a rural community of South-west Nigeria. Nigerian Journal of Clinical Practice 2016; 19 (2): 161-169.

4. Naicker S. End-stage renal disease in Sub-Saharan Africa. Ethn Dis. 2009; 19 (1 Suppl. 1): S1-13-5.

5. Arogundade FA, Sanusi AA, Hassan MO, Akinsola A. The pattern, clinical characteristics and outcome of ESRD in Ile-Ife, Nigeria: Is there a change in trend? Afr bealth Sci. 2011; 11(4): 594-601.

6. Coresh J, Selvin E, Stevens LA, Manzi J, Kusek JW, Eggers P, Van Lente F, Levey AS. Prevalence of chronic kidney disease in the United States. JAMA. 2007; 298 (17): 2038-2047.

7. Otero A, Gayoso P, Garcia F, de Francisco AL, EPIRCE study group. Epidemiology of chronic renal disease in the Galician population: Results of the pilot Spanich EPIRCE study. Kidney Int Suppl. 2005; 68 (Suppl 99): S16-S19.

8. Cirillo M, Laurenzi M, Mancini M, Zanchetti A, Lombardi C, De Santo NG. Low glomerular filtration in the population: Prevalence, associated disorders, and awareness. Kidney Int. 2006; 70 (4): 800-806.

9. Ulasi II, Ijoma CK. The enormity of chronic kidney disease in Nigeria: The situation in a teaching hospital in South-east Nigeria. J Trop Med. 2010; 2010: 501957.

10. Akokuwebe ME. Perceived susceptibility, morbidity and treatment patterns of kidney diseases among residents of Lagos State, Nigeria. A PhD thesis submitted to the Faculty of the Social Sciences, University of Ibadan, Nigeria. 2017; 160-170

11. Alebiosu CO, Ayodele OO, Abbas A, Olutoyin AI. Chronic renal failure at the Olabisi Onabanjo University Teaching Hospital, Sagamu, Nigeria. Afr Health Sci. 2006; 6 (3): 132-138.

12. Kadir S, Walker O, Salako BL, Akinkugbe O. Blood pressure, hypertension and correlates in urbanized workers in Ibadan, Nigeria: A revisit. J Hum Hypertens. 1999; 13(1): 23-27.

13. Schena FP. Epidemiology of end-stage renal disease: International comparisons of renal replacement therapy. Kidney Int. 2000; 57 (74): 39-45.
14. Egbi OG, Okafor UH, Miebodei KE, Kasia BE, Kunle-Olowu OE, Unuigbe EI. Prevalence and correlates of chronic kidney disease among civil servants in Bayelsa State, Nigeria. Nigerian Journal of Clinical Practice. 2014; 17 (5): 602-607.

15. Odubanjo MO, Oluwasola AO, Kadir S. The epidemiology of end-stage renal disease in Nigeria: The way forward. Int Urol Nephrol. 2011; 43 (3): 785-792.

16. Ulasi II, Ijoma CK, Onodugo OB, Arodiwe EB, Ifebunandu NA, Okoye JU. Towards prevention of: A community based study in South East Nigeria. Kidney Int Suppl. 2013; 3 (2): 195-201.

17. Oluyombo R, Ayodele OE, Akinwusi PO, Okunola OO, Akinsola A, Arogundade FA, Sanusi AA, Onayade A. A community of the prevalence, risk factors and pattern of chronic kidney disease in Osun State, South West Nigeria. West Afr J Med. 2013; 32 (2): 85-92.

18. Afolabi MO, Abioye-Kuteyi EA, Arogundade FA, Bello IS. Prevalence of chronic kidney disease in a Nigerian family practice population. SA Fam Pract 2009; 51 (2): 132-137.

19. Bamgboye EL. The looming epidemic of kidney failure in Nigeria. The magnitude of the problem. 2015 Alumni Session II. Consultant Transplant Nephrologist Clinical Director, St. Nicholas Hospital Lagos. President, Transplant Association of Nigeria President, Nigerian Association of Nephrology Director-MetroHealth HMO. 2015; 1-34.

20. Oluyombo R, Olamoyegun MA, Ayodele OE, Akinwusi PO, Akinsola A. Clustering of chronic kidney disease and cardiovascular risk factors in South-west Nigeria. J Nephrol Pathol. 2017; 6 (3): 196-203.

21. Kurella TM, Anand S, Li S, Chen SC, Whaley-Connell AT, Stevens LA, Norris KC. Comparison of CKD awareness in a screening population using the Modification of Diet in Renal Disease (MDRD) study and CKD Epidemiology Collaboration (CKD-EPI) equations. Am J Kidney Dis. 2011; 57 (3 Suppl 2): 17-23.

22. National Population Commission (NPC). Population distribution by sex, state, LGAs and Senatorial district: 2006 Census Priority Tables (vol. 3). National Population Commission, Abuja: Nigeria. Accessed November 3rd, 2016 from https://www.popualtion.gov. ng.

23. Kish L. A procedure for objective respondent selection within the household. Journal of the American Statistical Association. 1949; 380-387.

24. Amarya S, Singh K, Sabharwal M. health consequences of obesity in the elderly. Journal of Clinical Gerontology \& Geriatrics. 2014; 5 (3): 63-67.

25. Agaba EL, Agaba PA, Wigwe CM. Use and abuse of 
analgesics in Nigeria: A community survey. Niger J Med. 2004; 13 (4): 379-382.

26. Wilson D, Wakefield M, Owen N, Roberts L. Characteristics of heavy smokers. Prev. Med. 1992; 21 (3): 311-319.

27. Ekrikpo UE, Udo AI, Ikpeme EE, Effa EE. Haemodialysis in an emerging centre in a developing country: A two year review and predictors of mortality. $B M C$ Nephrol 2011; 12: 50.

28. Plantinga LC, Boulware LE, Coresh J, Stevens LA, Miller ER 3rd, Saran R, Messer KL, Levey AS, Powe NR. Patient awareness of chronic kidney disease: trends and predictors. Arch Interm Med. 2008; 168 (20): 22682275.

29. Boulware EL, Carson KA, Troll UM, Power NR, Cooper LA. Perceived susceptibility to chronic kidney disease among high-risk patients seen in primary care practices. Journal of General Internal Medicine. 2009; 24 (10): 1123-1129.

30. Adelekun TA, Ekwere TR, Akinsola A. The pattern of acute toxic nephropathy in Ife, Nigeria. West Afri J Med. 1999; 18 (1): 60-63.

31. Chen J, Wildman RP, Gu D, Kusek JW, Spruill M, Reynolds K, Liu D, Hamm LL, Whelton PK, He J. Prevalence of decreased kidney function in Chinese adults aged 35 to 74 years. Kidney Int. 2005; 68 (6): 2837-2845. 32. Viktorsdottir O, Palsson R, Andresdottir MB, Aspelund T, Gudnason V, Indridason OS. Prevalence of chronic kidney disease based on estimated glomerular filtration rate and proteinuria in Icelandic adults. Nephrol Dial Transplant. 2005; 20 (9): 1799-1807.

33. Minutolo R, De Nicola L, Mazzaglia G, Postorino
M, Cricelli C, Mantovani LG et al. Detection and awareness of moderate to advanced CKD by primary care practitioners: A cross-sectional study from Italy. Am J Kidney Dis. 2008; 52 (3): 444-453.

34. Ingsathit A, Thakkinstian A, Chaiprasert A, Sangthawan P, Gojaseni P. Prevalence and risk factors of chronic kidney disease in the Thai adult population: Thai SEEK study. Aephrol Dial Transplant. 2010; 25 (5): 1567-1575.

35. Nagamani S, Perumal SM, Perumal RLS, Kesavan C, Muthusamy K. ACE DD genotype associated with the female chronic kidney disease patients of Tamilnadu population. The Egyptian Journal of Medical Human Genetics. 2015; 16 (1): 29-33.

36. Kim S, Lim CS, Han DC, Kim GS, Chin HJ, Kim SJ, Cho WY, Kim YH, Kim YS. The prevalence of chronic kidney disease (CKD) and the associated factors to CKD in urban Korea: A population-based cross-sectional epidemiologic study. J Korean Med Sci. 2009; 24 (Suppl 1): S11-S21.

37. Rosenthal-Asher D, Maxwell L, Dover W, Sutton S, Cukor D. perceived risk of kidney diseases and its risk factors: results of a community health survey. Nephrol and Renal Dis. 2016; 1 (12): 35-37.

38. Bisi-Onyemaechi AI, Okafor HU, Ughasoro MD. Profile of chronic kidney disease modifiable risk factors in a rural community of South east Nigeria. BMC Public Health. 2018; 18 (1): 922.

39. Okwuonu CG, Chukwuonye II, Adejumo OA, Agaba EI, Ojogwu LI. Prevalence of chronic kidney disease and its risk factors among adults in a semi-urban community of South-East Nigeria. Niger Postgrad Med J. 2017; 24 (2): 81-87. 